\title{
Mechanisms of microRNA turnover
}

Maryam Sanei ${ }^{1}$ and Xuemei Chen ${ }^{1,2 *}$

${ }^{1}$ Department of Botany and Plant Sciences, Institute of Integrative Genome Biology, University of California, Riverside, CA

${ }^{2}$ Howard Hughes Medical Institute, University of California, Riverside, CA

*Corresponding author: xuemei.chen@ucr.edu 


\section{Abstract}

MicroRNAs (miRNAs) are 20-24 nucleotide (nt) RNAs that regulate gene expression by guiding Argonaute (AGO) proteins to specific target RNAs to cause their degradation or translational repression. The abundance of miRNAs is strictly controlled at the transcriptional or post-transcriptional levels. miRNA turnover is presumably a necessary means to regulate miRNA levels in response to physiological, developmental, and environmental changes. miRNA 3' end methylation, 3' end nucleotide addition, AGO and complementary target transcripts are known or probable processes/factors that affect miRNA stability and turnover. Here we discuss the mechanisms that control miRNA turnover in plants and, where applicable, make references to similarities and differences in these mechanisms between plants and animals.

\section{The biogenesis of miRNAs}

Regulation of gene expression by miRNAs helps to achieve the proper levels as well as the spatial and temporal patterns necessary for the biological functions of target genes. As regulatory molecules, miRNAs are known to impact plant and animal growth, development, metabolism, defense, and stress responses. Aberrant expression of miRNAs has been linked to many diseases in humans [1-3] and severe developmental abnormalities in Arabidopsis [4], and mice lacking miRNAs can not survive [5]. In plants, RNA polymerase II (Pol II) transcribes miRNA genes $(M I R)$ to result in pri-miRNAs that are 5' capped and 3' polyadenylated (miRNA biogenesis is reviewed in [6-8]). A primiRNA is cleaved at least twice by DICER-LIKE1 (DCL1) to form first the pre-miRNA and then the miRNA/miRNA* duplex. The duplex is methylated on the 3 ' ends by HUA ENHANCER1 (HEN1). The miRNA strand is selectively loaded into ARGONAUTE1 (AGO1) to form the miRNA-Induced Silencing Complex (miRISC), and the passenger strand (miRNA*) is usually degraded. In plants, an miRISC targets specific mRNAs for cleavage or translational repression (Fig 1). 
The widespread roles of miRNAs in gene regulation suggest that miRNAs themselves need to be tightly regulated. The steady-state levels of miRNAs can be controlled at multiple levels, such as transcription of MIR genes, processing of precursors, and turnover of mature miRNAs. Transcriptional regulation of MIR genes, like that of protein coding genes, relies on transcription factors and promoter activity. The TATA box [9] and many transcription factor binding motifs are present in the promoters of MIR genes in Arabidopsis [10-12]. Several transcription factors have been implicated or shown to influence MIR expression through binding sites in MIR promoters [10,11,13-15]. In plants, conserved miRNAs tend to belong to gene families with more than one member such that identical or highly similar miRNAs can be produced from different MIR genes. MIR family members can differ considerably in gene structure and regulatory sequences and undergo transcriptional regulation differently in response to environmental changes or developmental cues $[9,11,13,15-19]$.

The biogenesis of miRNAs is regulated at the global level through feedback on DCL1 and AGO1, critical players in miRNA biogenesis. The expression of DCL1 and AGO1 is repressed by two miRNAs, miR162 and miR168, respectively [20,21]. Feedback control by miR168 is necessary as Arabidopsis expressing miR168-resistant AGO1 exhibits developmental defects [22].

Knowing that miRNAs are on average ten times more stable than mRNAs [23] and that there may be needs for rapid changes in miRNA levels in biological settings makes the turnover of mature miRNAs a potentially important way of miRNA regulation. In this review, we summarize recent progress in the research on miRNA stability and turnover.

\section{3' methylation protects miRNAs from degradation}

5' cap structures and 3' polyA tails are added to most protein-coding Pol II transcripts to protect them from degradation. Unprotected $5^{\prime}$ or $3^{\prime}$ ends leave mRNAs vulnerable to exonucleolytic decay pathways. While pri-miRNAs are capped and polyadenylated, 
mature miRNAs lack any 3' poly A or 5' cap structures. 3' methylation protects nearly all miRNAs in plants from degradation. HEN1 proteins methylate the 3' ends of miRNAs and siRNAs in plants [24,25] and some siRNAs and nearly all PIWI-interacting RNAs (piRNAs) in animals [26-31]. The 2'-O-methyl mark protects small RNAs from 3' end uridylation and 3' $>$ ' exonucleolytic degradation [32]. The HEN1 protein in Arabidopsis consists of five structural domains, four of which directly interact with the small RNA substrate: a methyltransferase domain (MTase), two double-stranded RNA binding domains (dsRBD1 and dsRBD2) and a La-motif containing domain (LCD) that binds specifically to the 3' protruding end of the small RNA duplex. Arabidopsis HEN1 prefers to act on short double-stranded RNAs (19-25 nt) with 2 nt 3' overhangs [33]. Substrate length recognized by HEN1 is determined by the distance between the active site of the MTase domain and the $5^{\prime}$ end-capping site in the LCD [33].

Arabidopsis HEN1 methylates double-stranded small RNAs before their loading into AGO proteins [24], but HEN1 homologues in animals lack any dsRBDs [34] and methylate single-stranded small RNAs bound by AGO or PIWI (a clade of AGO proteins) $[26,29]$. This is consistent with the fact that nearly all small RNAs are 2'-O-methylated in plants, regardless of the AGO proteins to which they bind, but in animals small RNA methylation depends on which AGO protein HEN1 interacts with. siRNAs associated with AGO2 in Drosophila [35] and piRNAs loaded into PIWI in nearly all animals are methylated [26-30,36]. In Caenorhabditis elegans, in addition to piRNAs, small RNAs loaded to ERGO-1 (a clade of AGO proteins), named 26G RNAs, are methylated as well [37]. In Tetrahymena, small RNAs that are $\sim 28-29 \mathrm{nt}$ in length and interact with the PIWI protein Twi1p, known as scnRNAs, are methylated by HEN1 [31].

HEN1 promotes miRNA accumulation in plants - miRNAs in hen1 mutant Arabidopsis and rice plants are less abundant than in wild type [32,38-40]. The reduced miRNA accumulation in hen 1 mutants is accompanied by 3 ' truncation and ' 3 ' non-templated nucleotide addition (with uridine being the predominant nucleotide added) [32]. Loss of function in HEN1 in Drosophila [26,29], zebra fish [30], C. elegans [37], Tetrahymena [31], and mouse [34] also results in loss of small RNA methylation and increased 3' 
truncation and/or tailing. Although Drosophila miRNAs are generally loaded into AGO1 and are not methylated, a small fraction of miRNAs is loaded into AGO2 and is methylated by HEN1. Loss of 3' methylation of these miRNAs in Drosophila leads to their tailing and trimming, indicating that methylation, although not occurring to many miRNAs, does have a stabilization effect on some animal miRNAs as it does on plant miRNAs $[29,35,41]$.

In Arabidopsis, miRNA 3' methylation by HEN1 occurs widely to nearly all miRNAs, with the exception of miR158 and perhaps miR319. The incomplete methylation of these miRNAs results in their 3 ' truncation and tailing in wild type $[42,43]$. miRNA methylation in Drosophila occurs in a developmentally regulated manner, with the methylation status and abundance of some miRNAs increasing during aging. Lack of miRNA methylation results in accelerated neurodegeneration and shorter life span [41]. These observations suggest that small RNA methylation is regulated by as yet unknown mechanisms.

\section{Non-templated nucleotide addition to miRNAs}

3' non-templated nucleotide addition to miRNAs (or miRNA tailing) is a common phenomenon in plants and animals. The two most common types of miRNA tailing are adenylation and uridylation, and are carried out by nucleotidyl transferases such as noncanonical PolyA Polymerases (PAPs) and Terminal Uridylyl Transferases (TUTases) [44]. Non-templated nucleotide addition can impact miRNA gene regulatory networks through the control of miRNA biogenesis or stability, or by affecting miRNA activity. In human cell lines, specific miRNAs undergo changes in their 3' nucleotide addition during differentiation, indicating that 3 ' nucleotide addition is a biologically regulated process [45].

\section{miRNA adenylation}

Adenylation seems to stabilize miRNAs. In cotton wood, a significant portion of miRNAs was found to contain one or a few post-transcriptionally added adenylic acid residues at their 3' ends, and adenylated miRNAs were degraded slower in plant extracts in vitro [46]. Similarly, the addition of a single adenine to some miRNAs in animals appears to 
stabilize the miRNAs [47,48]. GLD2 (also known as TUT2, PAPD4 or HS1 in other organisms) was found to adenylate miRNAs and its depletion caused a reduction in miRNA accumulation [47,48]. 3' adenylation can also affect miRNA activity without affecting their stability. In humans, a small fraction of miRNAs sometimes undergoes adenylation after DICER processing but before RISC assembly. This adenylation is believed to interfere with RISC assembly and modulate miRNA targeting effectiveness $[49,50]$.

\section{miRNA uridylation}

Mature miRNAs can be uridylated in different species. In Chlamydomonas, MUT68 uridylates the $3^{\prime}$ termini of miRNAs and stimulates their degradation. MUT68 loss of function results in the accumulation of miRNAs in vivo [51]. In Arabidopsis, a MUT68 homologue HEN1 SUPPRESSOR1 (HESO1) is a terminal nucleotidyl transferase that adds non-templated uridine to the $3^{\prime}$ end of unmethylated miRNAs. HESO1 overexpression in a hen1 background causes reduced miRNA accumulation and more severe morphological defects [52]. Mutations in heso1 (including a null allele) reduce miRNA uridylation in hen1 mutant backgrounds, increase miRNA accumulation, and partially rescue the morphological phenotypes of hen1 [43,52]. This indicates that HESO1-mediated uridylation leads to miRNA degradation. The residual miRNA uridylation and incomplete rescue of hen1's morphological phenotypes by loss of function in HESO1 are attributable to another nucleotidyl transferase URT1 [53-55]. Both HESO1 and URT1 act on unmethylated miRNAs - their activities are completely inhibited by 3 ' methylation on the substrate miRNA $[43,52,54,55]$. Therefore, they may not play a role in miRNA turnover in the wild-type background in which most miRNAs are methylated. It remains to be determined whether other nucleotidyl transferases (there are a total of ten such potential enzymes in Arabidopsis) affect the turnover of methylated miRNAs. In humans, uridylation also has a general, negative effect on miRNA abundance by causing miRNA degradation [56]. Selective miRNA uridylation has been documented to play a role in development [57]. 
Uridylation may also affect the activities of miRNAs. In human cancer cells, terminal uridylation of miR26 by Zcchc11 appears to decrease its ability to repress its target genes without affecting its abundance [58]. In an Arabidopsis hen1 background, uridylation of miR158 by URT1 also affects the target repression activity of this miRNA without affecting its abundance [55]. In addition, uridylation of AGO1-bound miR165/6 by URT1 in vitro greatly reduces the slicing activity of miR165/6-AGO1 on its target RNA [55]. Intriguingly, monouridylation of miR171 in Arabidopsis hen1 backgrounds to result in a $22 \mathrm{nt}$ miRNA endows this miRNA the ability to trigger the biogenesis of secondary, phased siRNAs (also known as phasiRNAs) from its target mRNA [42]. Although $22 \mathrm{nt}$ miRNAs tend to trigger the production of phasiRNAs [59,60], miR171 is the exceptional miRNA that gains this ability in hen1 backgrounds, despite the fact that many miRNAs have $22 \mathrm{nt}$ forms in hen1 backgrounds.

\section{Nucleases involved in miRNA degradation}

Several exonucleases have been found to participate in miRNA degradation. SDN (SMALL RNA DEGRADING NUCLEASE) is a family of 3' $>5$ ' exonucleases that degrades mature miRNAs in Arabidopsis. Knockdown of three SDN family members (SDN1, 2, and 3) results in elevated levels of endogenous miRNAs and developmental defects [61]. SDN1 prefers single-stranded, non-uridylated miRNA substrates and its activity is partially inhibited by miRNA methylation [61]. As knocking down three SDN family members in the wild-type background, in which miRNAs are mostly methylated, results in higher levels of miRNAs, the SDN proteins must be able to degrade methylated miRNAs in vivo, as it does in vitro [61]. In C. elegans, the 5'->3' exonuclease XRN-2 degrades mature miRNAs in vitro and $x r n-2$ mutant worms over-accumulate some but not all miRNAs [62]. The Arabidopsis genome encodes three XRN enzymes. XRN4 degrades the $3^{\prime}$ fragments of miRISC-cleaved mRNAs and functions in general mRNA turnover, by means of degrading de-capped mRNAs [63,64]. XRN2 and XRN3 act in the process of miRNA biogenesis and digest the loops resulting from miRNA precursor processing [65]. $x r n 3$ and $x r n 2$ mutants exhibit strong developmental defects and accumulate high levels of miRNA loops [65]. There is as yet no evidence for a $5^{\prime}->3^{\prime}$ exonuclease activity acting on mature miRNAs in plants. 
The fact that uridylation promotes miRNA degradation implicates the existence of an enzyme that prefers uridylated miRNAs over non-uridylated miRNAs. This enzyme is unlikely SDN1 in Arabidopsis, as SDN1 is unable to degrade uridylated miRNAs in vitro [61]. In mammals, DIS3-like exonuclease 2 (DIS3L2), a 3' $>$ ' ' exonuclease belonging to the RNase II family, degrades oligouridylated pre-let-7 in vivo [66,67]. DIS3L2 also acts in the decay of mRNAs in mammals and yeast [68-70]. It exhibits tighter binding and higher activity on uridylated than non-uridylated substrates [68]. This suggests that DIS3L2 is a general RNA 3'->5' exonuclease that degrades uridylated RNAs. In Chlamydomonas, Ribosomal RNA-Processing protein 6 (RRP6), an exosome subunit, degrades uridylated small RNAs in vitro and RRP6 knockdown leads to increased accumulation of small RNAs in vivo [51]. In Arabidopsis, Suppressor Of Varicose (SOV), an orthologue of DIS3L2 in mammals, and RRP6 (there are three RRP6 genes) are candidates for the degradation of uridylated miRNAs.

\section{AG01 influences miRNA 3' modifications and stability}

AGO proteins are key players in miRNA biogenesis and function. Besides serving as an effector of miRNAs' activities, they protect and stabilize miRNAs from degradation in plants and animals [22,71]. In mutants in AGO1, the major miRNA effector in Arabidopsis, many miRNAs are reduced in abundance [22]. Loss of function in mammalian AGO2 significantly reduces miRNA stability, while overexpression of AGO2 prevents the degradation of miRNAs and increases miRNA half-life [72]. AGO's function in miRNA stability is independent of its endonuclease activity, as slicing-deficient mutants in mammalian AGO2 can still increase miRNA abundance [71]. A fraction of miR165/166 in plants is bound by AGO10, but AGO10 loss of function surprisingly increases the abundance of miR165/miR166 [73], which indicates that not all AGO proteins stabilize their associated miRNAs.

AGO proteins consist of four distinct domains: the N-terminal, PAZ, MID and PIWI (reviewed in [74]). The PAZ domain contains a specific binding pocket for the 3 ' end of a small RNA and the MID domain binds the 5' phosphate of a small RNA. Thus, AGO presumably protects both ends from degradation by making the ends inaccessible to 
nucleases. After the loading of miRNA/miRNA* to AGO, miRNA* is selectively degraded while the guide miRNA strand is bound by AGO. The disparate levels of miRNA and miRNA* strands in vivo support the protective role of AGO towards its associated small RNAs and indicates the presence of an efficient small RNA decay system that degrades unprotected small RNA strands [75].

Given the protective role of Arabidopsis AGO1 towards its associated miRNAs, it is counter-intuitive that miRNA 3' truncation and ' ' tailing require AGO1 in vivo. This was suggested by the observation that the partial loss of function ago1 allele, ago1-11, suppresses the 3' truncation and 3' tailing of miRNAs in a hen1 background [42]. Reciprocal co-immunoprecipitation experiments show that HESO1 interacts with AGO1 in an RNA-independent manner [76]. URT1 also interacts with AGO1 in vivo [54], and both HESO1 and URT1 can uridylate AGO1-bound miRNAs in vitro [55,76]. The interactions between AGO1 and HESO1 or URT1 probably serve two purposes. First, AGO1 probably recruits HESO1 to miRNA target transcripts to uridylate the 5' mRNA fragments generated by miRISC-mediated cleavage; this uridylation triggers the further degradation of these 5' mRNA fragments [76]. Second, AGO1 recruits HESO1 and URT1 to probably ensure the degradation of damaged or even intact miRNAs that need to be eliminated. In hen1 heso1 urt1 or heso1 urt1, 3' truncated miRNAs accumulate to higher levels, suggesting that HESO1 and URT1 eliminate shortened miRNAs or antagonize an exonulcease that trims miRNAs [54].

\section{Target transcripts may influence miRNA stability}

Target mimicry was first found in Arabidopsis to have a regulatory effect on miRNA activity. A non-protein-coding transcript from the IPS1 (INDUCED BY PHOSPHATE STARVATION1) gene in Arabidopsis has a binding site for miR399. A three-nucleotide bulge between the 10th and 11th position of the miRNA in IPS1 RNA prevents miR399guided cleavage such that IPS1 RNA serves as a target mimic (TM) to sequester miR399 from its endogenous target RNA [77]. Bioinformatics analyses suggest that many miRNAs in plants could potentially be regulated by endogenous TMs $[78,79]$. Endogenous TM RNAs are predicted to originate not only from noncoding regions but 
also from annotated genes and intragenic sequences [78,79]. TM RNAs with varied expression in different tissues may contribute to miRNA regulation in spatially or temporally specific manners [78].

Artificial TMs have become a powerful tool for regulating miRNA activities in vivo $[79,80]$. In transgenic lines expressing various artificial TMs, it was noticed that the levels of the cognate miRNAs were reduced [78-80], suggesting that the TMs led to miRNA degradation. Short Tandem Target Mimic (STTM) is an effective TM strategy that employs an artificial, non-coding STTM transcript that harbors two miRNA binding sites separated by a linker $[81,82]$. Intriguingly, the accumulation of an STTM transcript in vivo correlates with reduced levels of the cognate miRNA, suggesting that STTM induces miRNA degradation. STTM-induced degradation of miR165/6 was shown to require SDN1 and SDN2 [81]. The observations that TMs lead to lower miRNA levels raise many questions - How do TMs trigger miRNA degradation? Do natural target transcripts that can be cleaved by miRISC trigger miRNA degradation?

The effects of TM transcripts on miRNA stability may result from miRISC structural changes induced by TM RNAs. Structural studies with Thermus thermophilus AGO and its guide DNA and target RNA [83] revealed that a target RNA with extensive complementarity to the guide induces the release of the $3^{\prime}$ end of the guide from the AGO PAZ domain, whereas a target RNA with a short stretch of sequence complementarity to the guide does not do so. If these observations can be extrapolated to eukaryotic AGO-miRNA-target interactions, it would suggest that a target transcript with a high degree of sequence complementarity to the miRNA could condition the accessibility of the miRNA 3' end to exonucleases or nucleotidyl transferases and thus influence miRNA stability. miRNAs in animals recognize target mRNAs through seed pairing [84]. Extensive 3' pairing of miRNAs to artificial target RNAs triggers miRNA 3'$>5$ ' truncation, 3' tailing and decay in Drosophila [35] and mammalian cells [85,86] (Fig 2). By contrast, plant miRNAs are nearly perfectly complementary to their target RNAs [7]. Recognition of target transcripts by plant miRISCs could dislodge miRNA 3' ends from the PAZ domain and render miRNAs susceptible to 3 ' truncation and tailing (Fig 2) - 
this perhaps necessitates the methylation of plant miRNAs to protect them from degradation. TM transcripts could accumulate to high levels in vivo as they cannot be cleaved by miRISCs and therefore are more effective in rendering the degradation of miRNAs than natural target transcripts. Note that TMs can induce miRNA degradation in the WT background in which miRNAs are mostly methylated. This suggests that mechanisms are in place to degrade methylated miRNAs. While there could be an unidentified enzyme that removes the methyl group, the 3 ' truncation by an exonuclease such as SDN1 could also effectively result in an unmethylated miRNA, which would be susceptible to 3 ' tailing by nucleotidyl transferases (Fig 2). It remains to be determined whether STTM-induced miRNA degradation requires nucleotidyl transferases such as HESO1 and URT1.

\section{Acknowledgement}

We thank Malihe Sanei for her assistance in producing the illustrations in this manuscript. Research in the Chen lab on small RNA turnover is supported by grants from the Gordon and Betty Moore Foundation (GBMF3046), National Institutes of Health (GM061146) and National Science Foundation (IOS-1340001).

\section{References}

1. Jiang Q, Hao Y, Wang G, Juan L, Zhang T, Teng M, Liu Y, Wang Y: Prioritization of disease microRNAs through a human phenome-microRNAome network. BMC Syst Biol 2010, 4 Suppl 1:S2.

2. Chen $X$, Yan GY: Semi-supervised learning for potential human microRNAdisease associations inference. Sci Rep 2014, 4:5501.

3. Calin GA, Croce CM: MicroRNA signatures in human cancers. Nat Rev Cancer 2006, 6:857-866.

4. Schauer SE, Jacobsen SE, Meinke DW, Ray A: DICER-LIKE1: blind men and elephants in Arabidopsis development. Trends Plant Sci 2002, 7:487-491.

5. Yang WJ, Yang DD, Na S, Sandusky GE, Zhang Q, Zhao G: Dicer is required for embryonic angiogenesis during mouse development. J Biol Chem 2005, 280:9330-9335.

6. Rogers K, Chen X: Biogenesis, turnover, and mode of action of plant microRNAs. Plant Cell 2013, 25:2383-2399.

7. Voinnet O: Origin, biogenesis, and activity of plant microRNAs. Cell 2009, 136:669-687. 
8. Axtell MJ, Westholm JO, Lai EC: Vive la différence: biogenesis and evolution of microRNAs in plants and animals. Genome Biol 2011, 12:221.

9. Xie Z, Allen E, Fahlgren N, Calamar A, Givan SA, Carrington JC: Expression of Arabidopsis MIRNA genes. Plant Physiol 2005, 138:2145-2154.

10. Megraw M, Baev V, Rusinov V, Jensen ST, Kalantidis $K$, Hatzigeorgiou AG: MicroRNA promoter element discovery in Arabidopsis. RNA 2006, 12:16121619.

11. Zhao $\mathrm{X}$, Zhang $\mathrm{H}$, Li L: Identification and analysis of the proximal promoters of microRNA genes in Arabidopsis. Genomics 2013, 101:187-194.

12. Kim YJ, Zheng B, Yu Y, Won SY, Mo B, Chen X: The role of Mediator in small and long noncoding RNA production in Arabidopsis thaliana. EMBO J 2011, 30:814-822.

13. Yumul RE, Kim YJ, Liu X, Wang R, Ding J, Xiao L, Chen X: POWERDRESS and diversified expression of the MIR172 gene family bolster the floral stem cell network. PLoS Genet 2013, 9:e1003218.

14. Yant L, Mathieu J, Dinh TT, Ott F, Lanz C, Wollmann H, Chen X, Schmid M: Orchestration of the floral transition and floral development in Arabidopsis by the bifunctional transcription factor APETALA2. Plant Cell 2010, 22:2156-2170.

15. Xue XY, Zhao B, Chao LM, Chen DY, Cui WR, Mao YB, Wang LJ, Chen XY: Interaction between two timing microRNAs controls trichome distribution in Arabidopsis. PLoS Genet 2014, 10:e1004266.

16. Kawashima CG, Yoshimoto N, Maruyama-Nakashita A, Tsuchiya YN, Saito K, Takahashi H, Dalmay T: Sulphur starvation induces the expression of microRNA-395 and one of its target genes but in different cell types. Plant $J$ 2009, 57:313-321.

17. Jiang D, Yin C, Yu A, Zhou X, Liang W, Yuan Z, Xu Y, Yu Q, Wen T, Zhang D: Duplication and expression analysis of multicopy miRNA gene family members in Arabidopsis and rice. Cell Res 2006, 16:507-518.

18. Meyers BC, Souret FF, Lu C, Green PJ: Sweating the small stuff: microRNA discovery in plants. Curr Opin Biotechnol 2006, 17:139-146.

19. Li A, Mao L: Evolution of plant microRNA gene families. Cell Res 2007, 17:212218.

20. Vaucheret $\mathrm{H}$, Mallory AC, Bartel DP: AGO1 homeostasis entails coexpression of MIR168 and AGO1 and preferential stabilization of miR168 by AGO1. Mol Cell 2006, 22:129-136.

21. Xie Z, Kasschau KD, Carrington JC: Negative feedback regulation of Dicer-Like1 in Arabidopsis by microRNA-guided mRNA degradation. Curr Biol 2003, 13:784-789.

22. Vaucheret $H$, Vazquez $F$, Crete $P$, Bartel DP: The action of ARGONAUTE1 in the miRNA pathway and its regulation by the miRNA pathway are crucial for plant development. Genes Dev 2004, 18:1187-1197.

23. Gantier MP, McCoy CE, Rusinova I, Saulep D, Wang D, Xu D, Irving AT, Behlke MA, Hertzog PJ, Mackay F, et al.: Analysis of microRNA turnover in mammalian cells following Dicer1 ablation. Nucleic Acids Res 2011, 39:5692-5703. 
24. Yu B, Yang Z, Li J, Minakhina S, Yang M, Padgett RW, Steward R, Chen X: Methylation as a crucial step in plant microRNA biogenesis. Science 2005, 307:932-935.

25. Yang Z, Ebright $Y W, Y u$, Chen $X$ : HEN1 recognizes 21-24 nt small RNA duplexes and deposits a methyl group onto the 2' $\mathrm{OH}$ of the $3^{\prime}$ terminal nucleotide. Nucleic Acids Res 2006, 34:667-675.

26. Saito K, Sakaguchi Y, Suzuki T, Suzuki T, Siomi H, Siomi MC: Pimet, the Drosophila homolog of HEN1, mediates 2'-O-methylation of Piwi- interacting RNAs at their 3' ends. Genes Dev 2007, 21:1603-1608.

27. Kirino Y, Mourelatos Z: Mouse Piwi-interacting RNAs are 2'-O-methylated at their 3' termini. Nat Struct Mol Biol 2007, 14:347-348.

28. Ohara T, Sakaguchi Y, Suzuki T, Ueda H, Miyauchi K, Suzuki T: The 3' termini of mouse Piwi-interacting RNAs are 2'-O-methylated. Nat Struct Mol Biol 2007, 14:349-350.

29. Horwich MD, Li C, Matranga C, Vagin V, Farley G, Wang P, Zamore PD: The Drosophila RNA methyltransferase, DmHen1, modifies germline piRNAs and single-stranded siRNAs in RISC. Curr Biol 2007, 17:1265-1272.

30. Kamminga LM, Luteijn MJ, den Broeder MJ, Redl S, Kaaij LJ, Roovers EF, Ladurner $P$, Berezikov E, Ketting RF: Hen1 is required for oocyte development and piRNA stability in zebrafish. EMBO J 2010, 29:3688-3700.

31. Kurth HM, Mochizuki K: 2'-O-methylation stabilizes Piwi-associated small RNAs and ensures DNA elimination in Tetrahymena. RNA 2009, 15:675-685.

32. Li J, Yang Z, Yu B, Liu J, Chen X: Methylation protects miRNAs and siRNAs from a 3'-end uridylation activity in Arabidopsis. Curr Biol 2005, 15:1501-1507.

33. Huang $Y$, Ji L, Huang Q, Vassylyev DG, Chen X, Ma JB: Structural insights into mechanisms of the small RNA methyltransferase HEN1. Nature 2009, 461:823827.

34. Kirino $Y$, Mourelatos Z: The mouse homolog of HEN1 is a potential methylase for Piwi-interacting RNAs. RNA 2007, 13:1397-1401.

** 35. Ameres SL, Horwich MD, Hung JH, Xu J, Ghildiyal M, Weng Z, Zamore PD: Target RNA-directed trimming and tailing of small silencing RNAs. Science 2010, 328:1534-1539.

This study showed that an artificial target RNA with extensive complementarity to an AGO1-bound miRNA in the fruit fly triggers the tailing and trimming of the miRNA. This study reveals that the degree of target/miRNA complementarity influences miRNA stability.

36. Kirino $Y$, Mourelatos Z: 2'-O-methyl modification in mouse piRNAs and its methylase. Nucleic Acids Symp Ser (Oxf) 2007:417-418.

37. Billi AC, Alessi AF, Khivansara V, Han T, Freeberg M, Mitani S, Kim JK: The Caenorhabditis elegans HEN1 ortholog, HENN-1, methylates and stabilizes select subclasses of germline small RNAs. PLoS Genet 2012, 8:e1002617.

38. Boutet S, Vazquez F, Liu J, Beclin C, Fagard M, Gratias A, Morel JB, Crete P, Chen $\mathrm{X}$, Vaucheret $\mathrm{H}$ : Arabidopsis HEN1: a genetic link between endogenous miRNA controlling development and siRNA controlling transgene silencing and virus resistance. Curr Biol 2003, 13:843-848. 
39. Abe M, Yoshikawa T, Nosaka M, Sakakibara H, Sato $Y$, Nagato $Y$, Itoh J: WAVY LEAF1, an ortholog of Arabidopsis HEN1, regulates shoot development by maintaining microRNA and trans-acting small interfering RNA accumulation in rice. Plant Physiol 2010, 154:1335-1346.

40. Park W, Li J, Song R, Messing J, Chen X: CARPEL FACTORY, a Dicer homolog, and HEN1, a novel protein, act in microRNA metabolism in Arabidopsis thaliana. Curr Biol 2002, 12:1484-1495.

41. Abe M, Naqvi A, Hendriks GJ, Feltzin V, Zhu Y, Grigoriev A, Bonini NM: Impact of age-associated increase in 2'-O-methylation of miRNAs on aging and neurodegeneration in Drosophila. Genes Dev 2014, 28:44-57.

* 42. Zhai J, Zhao Y, Simon SA, Huang S, Petsch K, Arikit S, Pillay M, Ji L, Xie M, Cao $X$, et al.: Plant microRNAs display differential 3 ' truncation and tailing modifications that are ARGONAUTE1 dependent and conserved across species. Plant Cell 2013, 25:2417-2428.

This study demonstrated that miRNA trimming and tailing are AGO1 dependent, as an Arabidopsis ago1 hen1 double mutant shows reduced miRNA tailing or trimming. Additionally, the study examined miRNA 3' modifications in Arabidopsis, rice and maize hen 1 mutants and found that miRNA trimming prior to tailing is widespread among these species.

** 43. Zhao Y, Yu Y, Zhai J, Ramachandran V, Dinh TT, Meyers BC, Mo B, Chen X: The Arabidopsis nucleotidyl transferase HESO1 dylates unmethylated small RNAs to trigger their degradation. Curr Biol 2012, 22:689-694.

Along with [52], this paper identified HESO1 as one of the enzymes responsible for miRNA uridylation in Arabidopsis hen1 mutants and revealed that uridylation leads to miRNA degradation. It also showed that HESO1 is inactive on $3^{\prime}$ methylated miRNAs.

44. Martin G, Keller W: RNA-specific ribonucleotidyl transferases. RNA 2007, 13:1834-1849.

45. Wyman SK, Knouf EC, Parkin RK, Fritz BR, Lin DW, Dennis LM, Krouse MA, Webster PJ, Tewari M: Post-transcriptional generation of miRNA variants by multiple nucleotidyl transferases contributes to miRNA transcriptome complexity. Genome Res 2011, 21:1450-1461.

46. Lu S, Sun YH, Chiang VL: Adenylation of plant miRNAs. Nucleic Acids Res 2009, 37:1878-1885.

47. Katoh T, Sakaguchi Y, Miyauchi K, Suzuki T, Kashiwabara S, Baba T, Suzuki T: Selective stabilization of mammalian microRNAs by 3' adenylation mediated by the cytoplasmic poly(A) polymerase GLD-2. Genes Dev 2009, 23:433-438.

48. D'Ambrogio A, Gu W, Udagawa T, Mello CC, Richter JD: Specific miRNA stabilization by GId2-catalyzed monoadenylation. Cell Rep 2012, 2:1537-1545.

49. Burroughs AM, Ando Y, de Hoon MJ, Tomaru Y, Nishibu T, Ukekawa R, Funakoshi T, Kurokawa T, Suzuki H, Hayashizaki $Y$, et al.: A comprehensive survey of 3' animal miRNA modification events and a possible role for 3' adenylation in modulating miRNA targeting effectiveness. Genome Res 2010, 20:1398-1410. 
50. Nousch M, Yeroslaviz A, Habermann B, Eckmann CR: The cytoplasmic poly(A) polymerases GLD-2 and GLD-4 promote general gene expression via distinct mechanisms. Nucleic Acids Res 2014, 42:11622-11633.

* 51. Ibrahim F, Rymarquis LA, Kim EJ, Becker J, Balassa E, Green PJ, Cerutti H: Uridylation of mature miRNAs and siRNAs by the MUT68 nucleotidyltransferase promotes their degradation in Chlamydomonas. Proc Natl Acad Sci U S A 2010, 107:3906-3911.

This study concluded that MUT68 and RRP6 together degrade damaged miRNAs in miRNA quality control in Chlamydomonas. MUT68 uridylates miRNAs while RRP6 degradates uridylated miRNAs.

** 52. Ren G, Chen X, Yu B: Uridylation of miRNAs by HEN1 suppressor1 in Arabidopsis. Curr Biol 2012, 22:695-700.

This paper along with [43] showed that HESO1 is a terminal nucleotidyl transferase that uridylates unmethylated miRNAs and causes miRNA destabilization. The heso1 mutant partially rescues hen1's morphological phenotypes and HESO1 overexpression in hen1 causes more severe phenotypes.

53. Sement FM, Ferrier E, Zuber H, Merret R, Alioua M, Deragon JM, BousquetAntonelli $\mathrm{C}$, Lange $\mathrm{H}$, Gagliardi $\mathrm{D}$ : Uridylation prevents $3^{\prime}$ trimming of oligoadenylated mRNAs. Nucleic Acids Res 2013, 41:7115-7127.

54. Wang X, Zhang S, Dou Y, Zhang C, Chen X, Yu B, Ren G: Synergistic and independent actions of multiple terminal nucleotidyl transferases in the 3' tailing of small RNAs in Arabidopsis. PLoS Genet 11(4): e1005091.

55. Tu B, Liu L, Xu C, Zhai J, Li S, Lopez MA, Zhao Y, Yu Y, Ramachandran1 V, Ren $\mathrm{G}$, et al.: Distinct and cooperative activities of HESO1 and URT1 nucleotidyl transferases in microRNA turnover in Arabidopsis. PLOS Genet 11(4): e1005119.

56. Knouf EC, Wyman SK, Tewari M: The human TUT1 nucleotidyl transferase as a global regulator of microRNA abundance. PLoS One 2013, 8:e69630.

57. Thornton JE, Du P, Jing L, Sjekloca L, Lin S, Grossi E, Sliz P, Zon LI, Gregory RI: Selective microRNA uridylation by Zcchc6 (TUT7) and Zcchc11 (TUT4). Nucleic Acids Res 2014, 42:11777-11791.

58. Jones MR, Quinton LJ, Blahna MT, Neilson JR, Fu S, Ivanov AR, Wolf DA, Mizgerd JP: Zcchc11-dependent uridylation of microRNA directs cytokine expression. Nat Cell Biol 2009, 11:1157-1163.

59. Cuperus JT, Carbonell A, Fahlgren N, Garcia-Ruiz H, Burke RT, Takeda A, Sullivan CM, Gilbert SD, Montgomery TA, Carrington JC: Unique functionality of 22-nt miRNAs in triggering RDR6-dependent siRNA biogenesis from target transcripts in Arabidopsis. Nat Struct Mol Biol 2010, 17:997-1003.

60. Chen HM, Chen LT, Patel K, Li YH, Baulcombe DC, Wu SH: 22-Nucleotide RNAs trigger secondary siRNA biogenesis in plants. Proc Natl Acad Sci U S A 2010, 107:15269-15274.

** 61. Ramachandran $V$, Chen $X$ : Degradation of microRNAs by a family of exoribonucleases in Arabidopsis. Science 2008, 321:1490-1492. 
This is the first study reporting a family of exonucleases in the degradation of mature miRNAs. SDN1 acts on single-stranded miRNAs, is partially inhibited by the 2'-O-methyl group in the substrate miRNAs, and is inactive on urilydated miRNAs.

62. Chatterjee $\mathrm{S}$, Grosshans $\mathrm{H}$ : Active turnover modulates mature microRNA activity in Caenorhabditis elegans. Nature 2009, 461:546-549.

63. Souret FF, Kastenmayer JP, Green PJ: AtXRN4 degrades mRNA in Arabidopsis and its substrates include selected miRNA targets. Mol Cell 2004, 15:173-183.

64. Gazzani S, Lawrenson T, Woodward C, Headon D, Sablowski R: A link between mRNA turnover and RNA interference in Arabidopsis. Science 2004, 306:10461048.

65. Gy I, Gasciolli V, Lauressergues D, Morel JB, Gombert J, Proux F, Proux C, Vaucheret H, Mallory AC: Arabidopsis FIERY1, XRN2, and XRN3 are endogenous RNA silencing suppressors. Plant Cell 2007, 19:3451-3461.

66. Ustianenko D, Hrossova D, Potesil D, Chalupnikova K, Hrazdilova K, Pachernik J, Cetkovska K, Uldrijan S, Zdrahal Z, Vanacova S: Mammalian DIS3L2 exoribonuclease targets the uridylated precursors of let-7 miRNAs. RNA 2013, 19:1632-1638.

** 67. Chang HM, Triboulet R, Thornton JE, Gregory RI: A role for the Perlman syndrome exonuclease Dis3l2 in the Lin28-let-7 pathway. Nature 2013, 497:244-248.

This study identified DIS3L2 as an exoribonuclease that degrades uridylated prelet-7 in mouse. Knockdown of DIS3L2 causes the accumulation of uridylated prelet-7 in mouse embryonic stem cells.

68. Faehnle CR, Walleshauser J, Joshua-Tor L: Mechanism of Dis312 substrate recognition in the Lin28-let-7 pathway. Nature 2014, 514:252-256.

69. Malecki M, Viegas SC, Carneiro T, Golik P, Dressaire C, Ferreira MG, Arraiano CM: The exoribonuclease Dis3L2 defines a novel eukaryotic RNA degradation pathway. EMBO J 2013, 32:1842-1854.

70. Lubas M, Damgaard CK, Tomecki R, Cysewski D, Jensen TH, Dziembowski A: Exonuclease hDIS3L2 specifies an exosome-independent $3^{\prime}-5^{\prime}$ degradation pathway of human cytoplasmic mRNA. EMBO J 2013, 32:1855-1868.

71. Diederichs S, Haber DA: Dual role for argonautes in microRNA processing and posttranscriptional regulation of microRNA expression. Cell 2007, 131:10971108.

* 72. Winter J, Diederichs S: Argonaute proteins regulate microRNA stability: Increased microRNA abundance by Argonaute proteins is due to microRNA stabilization. RNA Biol 2011, 8:1149-1157.

This study measured the half-lives of miRNAs in mammlian cells and showed that AGO2 loss of function results in reduced miRNA half-lives while AGO2 overexpression increased miRNA half-lives. It was also shown that AGO2's role in stabilizing miRNAs is independent of its slicer activity. 
73. Liu $Q$, Yao $X$, Pi L, Wang $H$, Cui $X$, Huang $H$ : The ARGONAUTE10 gene modulates shoot apical meristem maintenance and establishment of leaf polarity by repressing miR165/166 in Arabidopsis. Plant $J$ 2009, 58:27-40.

74. Hutvagner G, Simard MJ: Argonaute proteins: key players in RNA silencing. Nat Rev Mol Cell Biol 2008, 9:22-32.

75. Kai ZS, Pasquinelli AE: MicroRNA assassins: factors that regulate the disappearance of miRNAs. Nat Struct Mol Biol 2010, 17:5-10.

* 76. Ren G, Xie M, Zhang S, Vinovskis C, Chen X, Yu B: Methylation protects microRNAs from an AG01-associated activity that uridylates 5' RNA fragments generated by AGO1 cleavage. Proc Natl Acad Sci U S A 2014, 111:6365-6370.

This study showed that HESO1 uridylates 5' mRNA fragments produced by miRISC-guided cleavage of miRNA target transcripts and triggers their degradation. The paper also reported that HESO1 interacts with AGO1 in Arabidopsis and proposed that miRNA $3^{\prime}$ methylation is necessary to protect miRNAs from uridylation by AGO1-associated HESO1.

77. Franco-Zorrilla JM, Valli A, Todesco M, Mateos I, Puga MI, Rubio-Somoza I, Leyva A, Weigel D, Garcia JA, Paz-Ares J: Target mimicry provides a new mechanism for regulation of microRNA activity. Nat Genet 2007, 39:1033-1037.

78. Wu HJ, Wang ZM, Wang M, Wang $\mathrm{XJ}$ : Widespread long noncoding RNAs as endogenous target mimics for microRNAs in plants. Plant Physiol 2013, 161:1875-1884.

79. Ivashuta S, Banks IR, Wiggins BE, Zhang Y, Ziegler TE, Roberts JK, Heck GR: Regulation of gene expression in plants through miRNA inactivation. PLOS One 2011, 6:e21330.

80. Todesco M, Rubio-Somoza I, Paz-Ares J, Weigel D: A collection of target mimics for comprehensive analysis of microRNA function in Arabidopsis thaliana. PLoS Genet 2010, 6:e1001031.

** 81. Yan J, Gu Y, Jia X, Kang W, Pan S, Tang X, Chen X, Tang G: Effective small RNA destruction by the expression of a short tandem target mimic in Arabidopsis. Plant Cell 2012, 24:415-427.

This study described a powerful new method named short tandem target mimic (STTM) to induce the degradation of specific miRNAs in vivo. STTMs are composed of two miRNA-binding sites that are linked by a short spacer. STTM-triggered degradation of miR165/6 was found to require SDN1 and SDN2.

82. Tang G, Yan J, Gu Y, Qiao M, Fan R, Mao Y, Tang X: Construction of short tandem target mimic (STTM) to block the functions of plant and animal microRNAs. Methods 2012, 58:118-125.

83. Wang Y, Juranek S, Li H, Sheng G, Tuschl T, Patel DJ: Structure of an argonaute silencing complex with a seed-containing guide DNA and target RNA duplex. Nature 2008, 456:921-926.

84. Bartel DP: MicroRNAs: target recognition and regulatory functions. Cell 2009, 136:215-233. 
85. Marcinowski L, Tanguy M, Krmpotic A, Radle B, Lisnic VJ, Tuddenham L, ChaneWoon-Ming B, Ruzsics Z, Erhard F, Benkartek C, et al.: Degradation of cellular mir-27 by a novel, highly abundant viral transcript is important for efficient virus replication in vivo. PLoS Pathog 2012, 8:e1002510.

86. Cazalla D, Yario T, Steitz JA: Down-regulation of a host microRNA by a Herpesvirus saimiri noncoding RNA. Science 2010, 328:1563-1566. 


\section{Figure Legends}

Figure 1. The miRNA biogenesis pathway in Arabidopsis. MIR genes are transcribed by Pol II to produce a long primary miRNA transcript (pri-miRNA). DCL1 processes the primiRNA to a miRNA/miRNA* duplex with a pre-miRNA intermediate. HEN1 stabilizes the duplex via 2'-O- methylation of the 3' ends. The miRNA strand is loaded selectively into AGO1, forming a miRISC that regulates miRNA target gene expression.

Figure 2. Target complementarity probably affects miRNA degradation in animals and plants. In animals, miRNA pairs with its target mRNA through the 5' seed region. The limited complementarity probably leaves the unmethylated miRNA 3' end protected inside the AGO protein. An artificial target RNA with extensive pairing to an miRNA leads to miRNA 3' trimming and 3' tailing by unknown enzymes, perhaps by dislodging the miRNA 3' end from AGO. In plants, miRNAs are nearly fully complementary to their targets and miRNA/target pairing may expose the miRNA $3^{\prime}$ end. In the absence of HEN1 activity, an unmethylated miRNA is subjected to 3' trimming and tailing by exonucleases (perhaps SDN1 being one of them) and nucleotidyl transferases (HESO1 and URT1), respectively. Enzymes involved in degrading tailed miRNAs are unknown. Although the 2'-O-methyl mark stabilizes plant miRNAs, methylated miRNAs can be degraded. The degradation of methylated miRNAs may entail demethylation by an unknown enzyme, or may be initiated by exonucleolytic trimming, which results in a 3' truncated and unmethylated miRNA. 


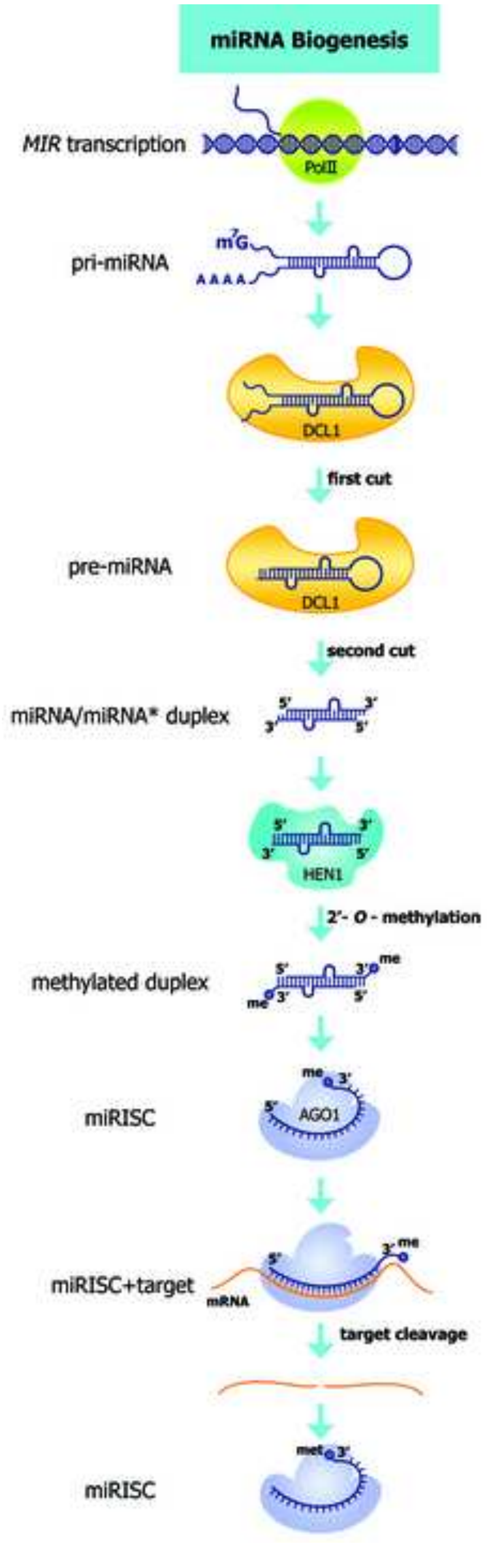

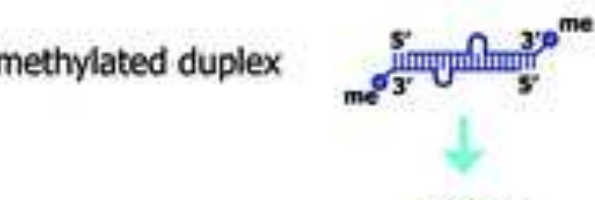

.

(2.
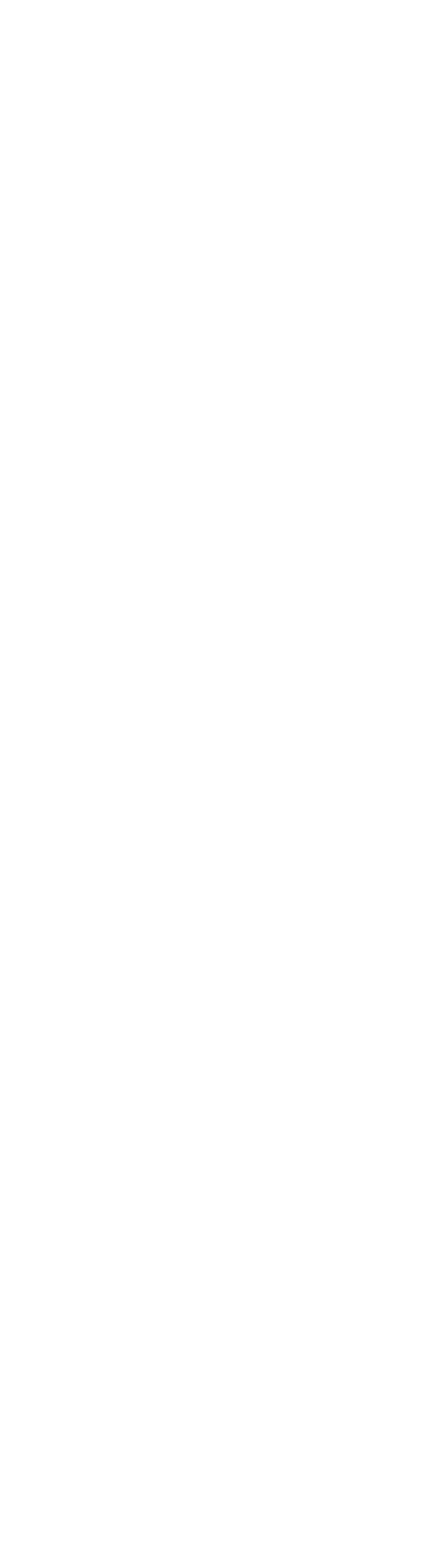


\section{miRNA turnover}
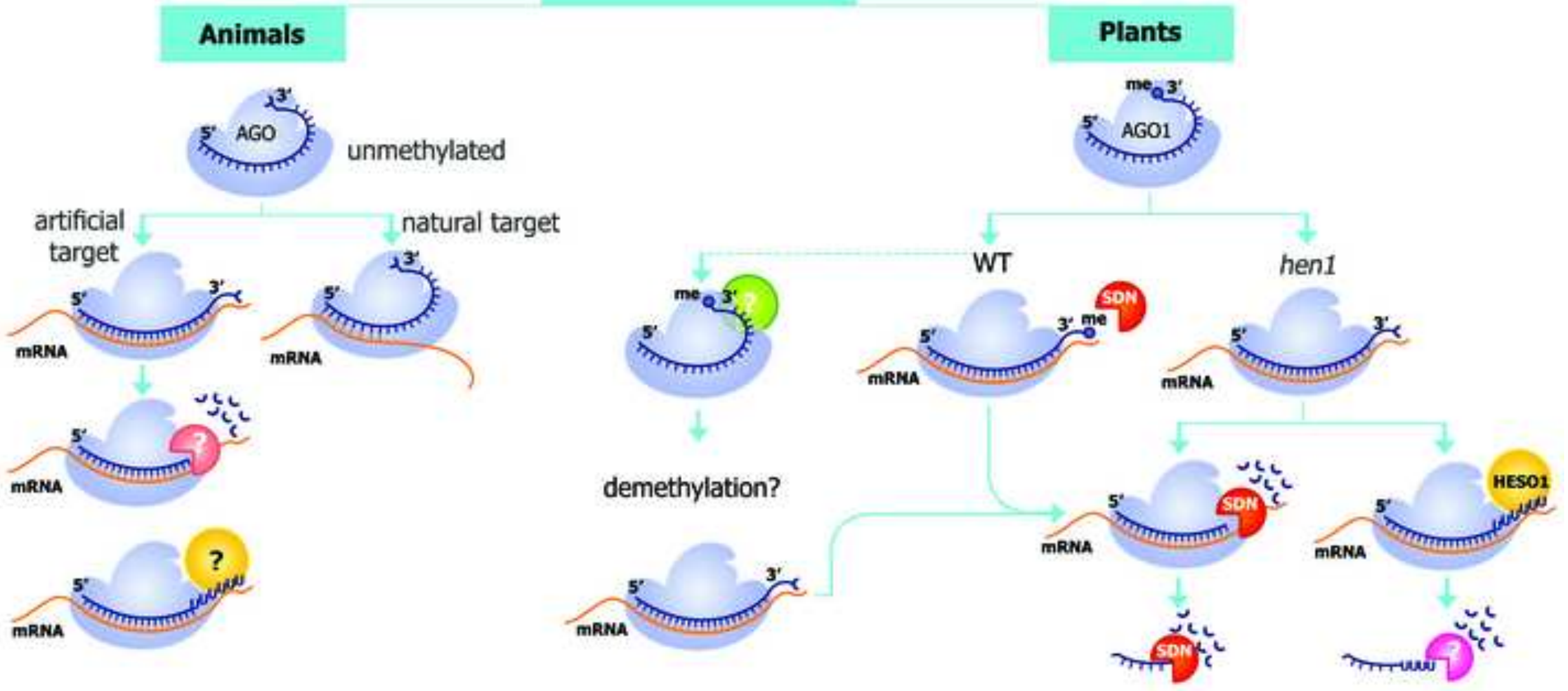